\title{
Prevalence, risk factors and prognosis of nontuberculous mycobacterial infection among people with bronchiectasis: a population survey
}

\author{
To the Editor:
}

The incidence of nontuberculous mycobacteria (NTM) infection among people with bronchiectasis varies between different geographical areas and accordingly between different series [1,2]. Studies are largely based on bronchiectasis referral centres, which routinely screen for NTM in respiratory secretions. Therefore, the reported estimates of NTM prevalence in bronchiectasis may be exaggerated. Studies from bronchiectasis centres show conflicting results regarding risk factors for NTM: older age was found to increase risk in some but not other centres [1-4]. Due to the small numbers of patients in these studies, it is usually not possible to determine the effect of NTM infection on prognosis.

Utilising the population registry of Israel's largest health maintenance organisation [5], we aimed to determine the incidence, risk factors and prognosis of NTM infection among patients with bronchiectasis.

The database was searched for adults with bronchiectasis on January 1,2010, excluding cystic fibrosis and idiopathic pulmonary fibrosis. We searched for laboratory codes indicating NTM between January 2010 and December 2016. We defined three categories of NTM. 1) "Growth": a single culture positive for any NTM. 2) "Colonisation": at least two cultures positive for the same NTM species. 3) "Treated": either of the above categories, treated with three or more antimycobacterial drugs. Medication use was determined from pharmacy reports. Socioeconomic status (SES) was based on the SES score of the clinic neighbourhood as defined by the Israeli Central Bureau of Statistics [6]. Mortality and hospitalisations during follow-up (2010-2016) were compared between patients with and without NTM infection.

Multivariate logistic regression was used to identify the NTM risk factors. The Cox time dependent model was used to identify prognostic factors that influence hospitalisation and mortality.

On January 1, 2010, the database included 2710432 adults. Of these, 6347 had a diagnosis of bronchiectasis (234 in 100000). 6274 patients were without NTM infection prior to January 1. Of them, only 1871 (30\%) patients had mycobacterial cultures available. In the general population (without bronchiectasis), $1476(0.055 \%)$ people had NTM from respiratory specimens during follow up. Among people with bronchiectasis ( $\mathrm{n}=6274), 105$ (1.7\%) grew NTM during follow-up. Only $30(0.48 \%)$ cases had two or more cultures of the same NTM species, representing colonisation. Treatment with a combination of three or more antimycobacterial drugs was found in $12(0.19 \%)$ patients.

The incidence of NTM growth among all patients with bronchiectasis $(n=6274)$ was estimated in four strata of age: $18-39,40-59,60-79$ and $\geqslant 80$ years. The corresponding incidence of NTM growth were $1.1 \%, 2.2 \%, 1.9 \%$ and $0.9 \%$, respectively $(\mathrm{p}=0.030) .50 \%$ of patients were $60-80$ years old with a similar age distribution between patients with and without available mycobacterial cultures. Overall, $43 \%$ of patients with bronchiectasis in our study had emigrated from areas where tuberculosis is common, specifically Africa and Eastern Europe. NTM incidence was 1.5\% in immigrants from Africa and Eastern Europe, and $1.8 \%$ in others $(\mathrm{p}=0.355)$.

In bronchiectasis, the most common NTM species were Mycobacterium simiae ( $\mathrm{n}=35,33 \%)$, Mycobacterium avium-intracellulare (MAI) (26, 25\%), Mycobacterium fortuitum ( $\mathrm{n}=16,15 \%)$ and socioeconomic status http://ow.ly/E3jR30j553X

Cite this article as: Shteinberg M, Stein N, Adir Y, et al. Prevalence, risk factors and prognosis of nontuberculous mycobacterial infection among people with bronchiectasis: a population survey. Eur Respir J 2018; 51: 1702469 [https://doi.org/10.1183/13993003.02469-2017]. 
Mycobacterium abscessus ( $\mathrm{n}=11,10.5 \%)$. Growth of $M$. abscessus and of $M$. simiae were significantly more common than in the general population ( $M$. abscessus: $5.5 \%, \mathrm{p}=0.035 ;$ M. simiae: $23.5 \%, \mathrm{p}=0.023$ ). Colonised patients had M. simiae, MAI (30\% each) and M. abscessus (27\%). Patients that were treated for NTM infection had MAI (50\%), M. abscessus (25\%) and Mycobacterium kansasii (25\%).

In a multivariate logistic regression model that included 5737 bronchiectasis patients with complete information, young age, female sex, having never smoked, medium or high SES, glucocorticoid use and antibiotic use were all independent risk factors for NTM growth. We repeated the model taking only the 1871 patients for whom mycobacterial cultures were available: nonsmoking and medium-high SES remained significantly associated with NTM growth. We compared the 30 patients with colonisation of NTM to the two other groups (patients with a single growth or no NTM growth). Younger age, female sex and having been treated with antibiotics or prednisolone were significantly associated with colonisation. A history of past smoking was significantly less common among patients with colonisation than patients with a single NTM growth $(\mathrm{p}=0.024)$ (table 1$)$.

Over the follow up period, there were 1697 deaths and 3959 hospitalizations among 5737 patients with complete information. Growth of NTM was associated with a higher risk of mortality (adjusted hazard ratio (HR) 1.56, 95\% CI 1.02-2.40; $\mathrm{p}=0.039$ ) and hospitalisations (HR 1.57, 95\% CI 1.05-2.34; $\mathrm{p}=0.029$ ). Among the 30 patients with colonisation of NTM, mortality was not increased compared to noncolonised patients (adjusted HR 1.13, 95\% CI 0.47-2.75; $\mathrm{p}=0.784$ ).

In this study, based on a database analysis of $>6000$ patients with bronchiectasis, we found that the incidence of NTM growth in bronchiectasis is considerably lower than previously reported: over 7 years of follow up, only $1.7 \%$ of the patients with bronchiectasis had detection of NTM species from respiratory infections, representing 5.6\% positive cultures (slightly less than previous reports $[1-4,7,8]$ ). $<0.48 \%$ had colonisation and only $0.19 \%$ were treated for NTM. An incidence of $1.7 \%$ may be an underestimation of the true incidence, since only $30 \%$ of patients had a mycobacterial culture sent. This figure may be a reflection of the practice (supported by guidelines) to send mycobacterial cultures only when there is a clinical suspicion of NTM pulmonary disease, rather than routine testing. Similarly to previously described in Israel [9], we found a high prevalence of $M$. simiae, with only a minority of species considered pathogenic. Interestingly, we did not find any isolation of Mycobacterium xenopi in bronchiectasis, and only $27(1.8 \%)$ in patients without bronchiectasis, unlike previously described results from Israel.

We could not determine how many of our patients filled American Thoracic Society/Infectious Diseases Society of America (ATS/IDSA) criteria for NTM lung infection [10]. However, many of the strains in our

TABLE 1 Demographic and clinical characteristics distribution according to nontuberculous mycobacteria (NTM) status (no NTM versus single growth versus colonisation) and adjusted odds ratios (95\% CI) for the association with any NTM growth (composite of single growth and colonisation)

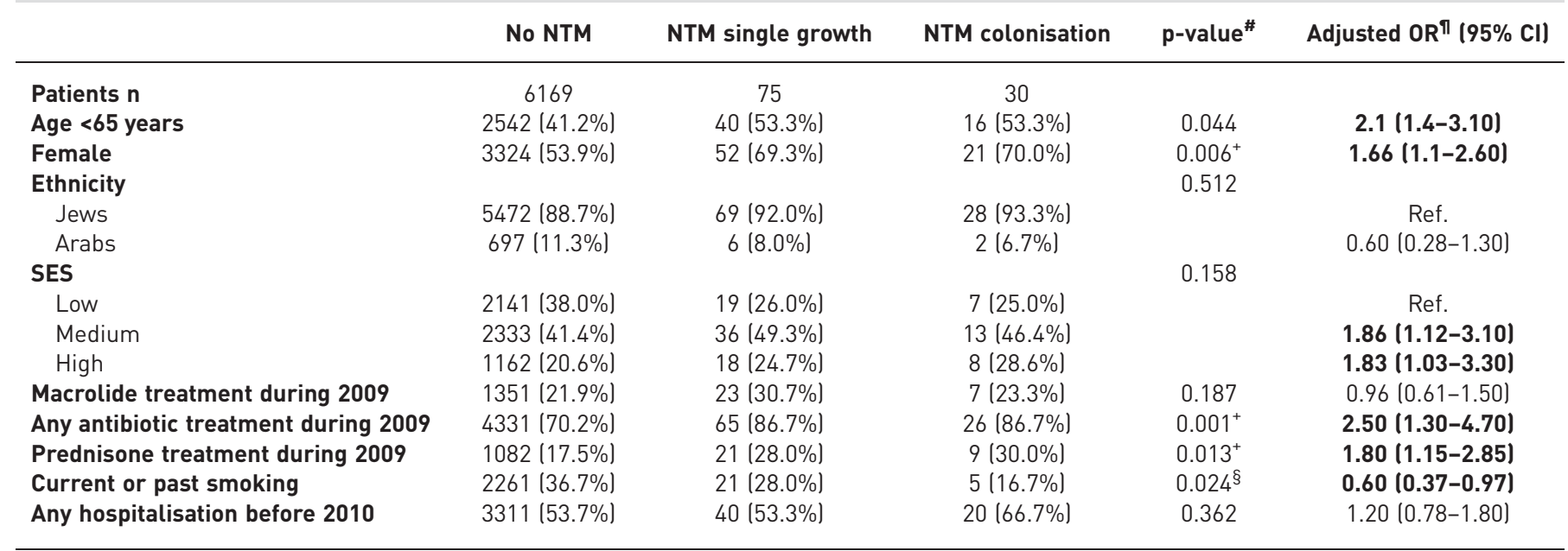

Data are presented as $\mathrm{n}(\%)$ unless otherwise stated. Risk assesment of acquiring NTM growth and colonisation. SES: socioeconomic status.

${ }^{\#}$ : comparison between three groups; П: adjusted odds ratio for any growth (composite of single growth and colonisation); ${ }^{+}$: significant difference after Bonferroni correction between no NTM and NTM single growth; ${ }^{\S}$ : significant difference after Bonferroni correction between NTM single growth and NTM colonisation. Bold indicates statistically significant odds ratios. 
population are not considered pathogenic or are resistant to antimicrobials [11], with M. simiae comprising $30 \%$ of the strains. Therefore, the decision to withhold treatment may have been justified.

A higher risk of NTM growth was found among patients with a high SES, never-smoking and indices of disease severity, such as hospitalisation and glucocorticoid and antibiotic treatment (table 1). This association may reflect surveillance bias, as these patients may be subject to intensive follow-up because of disease severity or increased health consciousness, leading to more frequent testing and detection of NTM. We achieved similar results when we repeated the analysis only in individuals with available mycobacterial cultures. Prior glucocorticoid and antibiotics use may increase the risk for NTM infection, respectively by immune compromise and selecting for resistant organisms.

We found that smoking was associated with a lower risk for NTM growth (28\% past or present smokers versus $36 \%$ among people without NTM growth), and even more so for colonisation $(16.7 \%, \mathrm{p}=0.024)$. A similar association was found previously in another bronchiectasis cohort [12], and has been reported in other granulomatous lung diseases such as sarcoidosis and hypersensitivity pneumonitis [13]. Inhibition of granuloma formation by smoking may possibly explain our finding [14]. Another factor associated with lower risk for NTM growth was low SES. A possible explanation is that smoking, which is more prevalent among people from a low SES, was not fully accounted for. Other factors associated with higher SES may also be important; possibly, use of home water filtering systems.

In our analysis, younger age was associated with an elevated risk of NTM growth, with the highest prevalence in the 40-59-years age group. Previous studies in bronchiectasis patients [4, 12, 15] were contradicting in the association between age and NTM growth.

Growth of NTM was associated with an increased risk of mortality (adjusted HR 1.56; p=0.039) and hospitalisations (adjusted HR 1.57; $\mathrm{p}=0.029$ ). In the 30 patients with colonisation, mortality was not increased.

Our study has several limitations. The variables were based on electronic medical records and may be subject to inaccuracy or underreporting. We could not assess whether individuals with NTM fulfilled ATS/ IDSA criteria for lung NTM infection [10]. We did not have data on radiological findings or lung function, nor could we calculate a bronchiectasis severity score, and we used three antimycobacterial drugs as a surrogate for NTM treatment. However, NTM lung disease in Israel is managed exclusively by pulmonologists, who are familiar with the NTM guidelines for diagnosis and treatment. Furthermore, the identification of NTM species is centralised by reference mycobacterial laboratories. In addition, this study included data from a large cohort of people with bronchiectasis and therefore could assess risk factors for NTM acquisition. Further research is required to explore the association of NTM with higher SES and younger age, and the negative association with smoking.

Michal Shteinberg ${ }^{1,2,3}$, Nili Stein ${ }^{3,4}$, Yochai Adir ${ }^{1,3}$, Shifra Ken-Dror ${ }^{5}$, David Shitrit ${ }^{6,7}$, Danielle Bendayan ${ }^{7,8}$, Leonardo Fuks ${ }^{7,9}$ and Walid Saliba ${ }^{3,4}$

${ }^{1}$ Pulmonology Institute, Carmel Medical Center, Haifa, Israel. ${ }^{2} \mathrm{CF}$ Center, Carmel Medical Center, Haifa, Israel. ${ }^{3}$ Technion - Israel Institute of Technology, B. Rappaport Faculty of Medicine, Haifa, Israel. ${ }^{4}$ Dept of Community Medicine and Epidemiology, Carmel Medical Center, Clalit Health Services, Haifa, Israel. ${ }^{5}$ Microbiology Laboratory, Haifa and Western Gallilee, Clalit Health Services, Haifa, Israel. ${ }^{6}$ Pulmonology Dept, Meir Medical Center, Kfar Sava, Israel. ${ }^{7}$ Sackler Faculty of Medicine, Tel Aviv University, Tel Aviv, Israel. ${ }^{8}$ Pulmonary and Tuberculosis Dept, Shmuel Harofe Hospital, Be’er Ya’akov, Israel. ${ }^{9}$ Pulmonary Institute, Rabin Medical Center, Petah Tikva, Israel.

Correspondence: Michal Shteinberg, Pulmonology Institute, Carmel Medical Center, 7 Michal St, Haifa 34362, Israel.

E-mail: michalsh@technion.ac.il

Received: Nov 172017 | Accepted after revision: Feb 262018

Acknowledgement: This work was presented at the second World Bronchiectasis Conference in July 2017 (Milan, Italy).

Conflict of interest: M. Shteinberg reports having received research grants from Novartis and Trudell Pharma, travel grants from Actelion, BI, GSK and Rafa, and speaker's fees from BI, GSK, AstraZeneca, Teva and Novartis, and is a member of the EMBARC collaboration. Y. Adir reports having received travel grants from Actelion, BI, GSK and Rafa, and speaker's fees from BI, GSK, AstraZeneca, Teva and Novartis.

\section{References}

1 Máiz L, Girón R, Olveira C, et al. Prevalence and factors associated with nontuberculous mycobacteria in non-cystic fibrosis bronchiectasis: a multicenter observational study. BMC Infect Dis 2016; 16: 437.

2 Aksamit TR, O'Donnell AE, Barker A, et al. Adult bronchiectasis patients: a first look at the United States Bronchiectasis Research Registry. Chest 2016; 151: 982-992.

3 Fowler SJ, French J, Screaton NJ, et al. Nontuberculous mycobacteria in bronchiectasis: prevalence and patient characteristics. Eur Respir J 2014; 28: 417-425.

4 Mirsaeidi M, Hadid W, Ericsoussi B, et al. Non-tuberculous mycobacterial disease is common in patients with non-cystic fibrosis bronchiectasis. Int J Infect Dis 2013; 17: e1000-4 
5 Saliba W, Barnett-Griness O, Rennert G. Red cell distribution width and all-cause mortality in patients with atrial fibrillation: a cohort study. J Arrhythmia 2017; 33: 56-62.

6 Burck L, Feinstein Y. Characterization and Classification of Geographical Units by the Socioeconomic Level of the Population. Jerusalem, Central Bureau of Statisics, 2000. Available from: www.cbs.gov.il/webpub/pub/text_page_ eng.html?publ $=100 \&$ CYear $=2008 \&$ CMonth $=1$.

7 Shteinberg M, Yaari N, Stein N, et al. [Bronchiectasis - review of literature and clinical characteristics of bronchiectasis patients treated in the bronchiectasis clinic at the Carmel Medical Center]. Harefuah 2015; 154: 356-361.

8 Izhakian S, Wasser WG, Fuks L, et al. Lobar distribution in non-cystic fibrosis bronchiectasis predicts bacteriologic pathogen treatment. Eur J Clin Microbiol Infect Dis 2016; 35: 791-796.

9 Braun E, Sprecher H, Davidson S, et al. Epidemiology and clinical significance of non-tuberculous mycobacteria isolated from pulmonary specimens. Int J Tuberc Lung Dis 2013; 17: 96-99.

10 Griffith DE, Aksamit T, Brown-Elliott BA, et al. An official ATS/IDSA statement: diagnosis, treatment, and prevention of nontuberculous mycobacterial diseases. Am J Respir Crit Care Med 2007; 175: 367-416.

11 van Ingen J. Microbiological diagnosis of nontuberculous mycobacterial pulmonary disease. Clin Chest Med 2015; 36: 43-54.

12 Wickremasinghe M, Ozerovitch LJ, Davies G, et al. Non-tuberculous mycobacteria in patients with bronchiectasis. Thorax 2005; 60: 1045-1051.

13 Blanchet M-R, Israël-Assayag E, Cormier Y. Inhibitory effect of nicotine on experimental hypersensitivity pneumonitis in vivo and in vitro. Am J Respir Crit Care Med 2004; 169: 903-909.

14 Maier L. Is smoking beneficial for granulomatous lung diseases? Am J Respir Crit Care Med 2004; 169: 893-894.

15 Fowler SJ, French J, Screaton NJ, et al. Nontuberculous mycobacteria in bronchiectasis: prevalence and patient characteristics. Eur Respir J 2006; 28: 1204-1210. 